\title{
Fundamentals of Inductively Coupled Wireless Power Transfer Systems
}

\author{
Ali Abdolkhani \\ Additional information is available at the end of the chapter
}

http://dx.doi.org/10.5772/63013

\begin{abstract}
The objective of this chapter is to study the fundamentals and operating principles of inductively coupled wireless power transfer (ICWPT) systems. This new technology can be used in various wireless power transfer applications with different specifications, necessities, and restrictions such as in electric vehicles and consumer electronics. A typical ICWPT system involves a loosely coupled magnetic coupling structure and power electronics circuitries as an integrated system. In this chapter, the emphasis is placed on the magnetic coupling structure, which is the mostimportant part of the system. Although this technology has motivated considerable research and development in the past two decades, still there are several theoretical studies such as the level of the operating frequency, operating at high secondary circuit quality factor, coupling efficiency, etc., that need further investigation to fully develop the governing mathematical relationships of this technology.

The chapter begins with an outline about the ICWPT systems highlighting their major application areas, followed by present challengesin the field. Then, theoperating principle of such a technology is presented, which includes system tuning, electrical equivalent circuit, powertransfercapability, and powerlossesassociated with thesystem. Thechapter ends with detailed derivations of the system coupling efficiency, which is the most important portion of the system efficiency analysis for both series- and parallel-tuned secondary side.
\end{abstract}

Keywords: Compensation, Coupling efficiency, Inductive power transfer, Magnetic coupling, Power transfer capability, Resonant converters, Wireless power transfer 


\section{Introduction}

Wireless power transfer is to transfer electrical power from one point to another through an air gap without any direct electrical contacts. This technology has been used for applications as in electric vehicles (EVs), consumer electronics, biomedical, etc. where conventional wires are inconvenient, hazardous, unwanted, or impossible. For instance, supplying electrical power using mechanical slip-rings in rotary applications results in a system with a reduced life span because of the frequent maintenance requirements due to wear and tear caused by friction. Moreover, mechanical slip-rings are associated with arcing, which make them unsafe to operate in the presence of explosive gases. Increasing the lifespan, reliability, and lowmaintenance operation can be achieved by eliminating the cables, mechanical slip-rings, as well as plugs and sockets.

In the early days of electromagnetism before the electrical-wire grid was deployed, serious interest and effort were devoted (most notably by Nikola Tesla) towards the development of schemes to transfer energy over long distances without any carrier medium. These efforts appear to have met with little success. Radiative modes of omni-directional antennas (which work very well for information transfer) are not suitable for power transfer, because a vast majority of power is wasted into free space. Today, we face a different challenge than Tesla. Now that the existing electrical-wire grids carry power almost everywhere, wireless power transfer technology creates new possibilities to supply portable devices with electrical energy, which has been used in many different applications. A range of applications has been considered and several approaches have been proposed based on the individual requirements. Currently, the main areas of wireless power transfer applications can be categorised as follows [1]:

- Industrial (operation in harsh environment, e.g. mining, next to explosive gases)

- Automotive (electric cars and general battery charging)

- Aerospace (transferring energy to moving parts)

- Consumer electronics (charging a cell phone or a laptop wirelessly)

- Biomedical (inductive interface to power implantable biomedical devices)

\section{Present challenges}

\subsection{Theoretical developments}

The aim of an inductively coupled wireless power transfer (ICWPT) system is to provide power to a movable object across a gapped magnetic structure. Its theoretical development relies on both magnetic and power electronics together as an integrated system. In the case of magnetic structure, designing a magnetic coupling structure with a small air gap would result in high magnetic coupling coefficient and increased power transfer capability. Modelling and 
representing the magnetic circuit and associate its geometrical characteristics with its electrical behaviour are very important as: (1) to enable predicting the circuit performance and (2) to provide the insight needed to achieve an optimised design. Furthermore, the magnetic structure of an ICWPT system combines the magnetic properties of both an ideal transformer and an inductor. There are more room for theoretical improvements in magnetisation, mutual inductance, leakage inductance, and their connection with the structure geometry and AC losses that are critical in power electronic designs. In ICWPT systems, in order to reduce the skin and proximity effects associated with the coils, multi-strands-woven Litz wire is often used. Modelling and developing functional analysis of such a phenomenon associated with Litz wire are of high importance for the development of an efficient ICWPT system.

Power electronics, on the other hand, covers a large area including electronics, control, and communications. Analysis and modelling of switch-mode non-linear circuits are the main concerns. Like most other power electronic applications, the further development of ICWPT systems depends largely on some fundamental advances in switch-mode non-linear theories. Moreover, the loose magnetic coupling between the primary and the secondary coils of an ICWPT power supply is more difficult to analyse than a traditional closely coupled transformer. This further increases the circuit complexity so that proper compensation and control have to be taken into consideration in the design [2].

\subsection{Technical limitations}

Because of the air gap, designing an ICWPT system poses some unusual design constraints compared to the traditional compactly coupled design. The relatively large gap in the magnetic circuit results in a low mutual inductance and high leakage inductances. Eddy currents caused by fringing flux can be formed in the magnetic material near the air gap and cause power losses and EMI. Operating at high frequencies presents unique design problems due to the increased core losses, leakage inductance, and winding capacitance. This is because physical orientation and spacing of the windings determine the leakage inductance and winding capacitance which are distributed throughout the windings in the magnetic structure [3]. Experienced SMPS (switch-mode power supply) designers know that SMPS success or failure heavily depends on the proper design and implementation of the magnetic components. Inherent parasitic elements in high-frequency inductive power transfer applications cause a variety of circuit problems including: high power losses, high-voltage spikes necessitating snubbers or clamps, poor cross regulation between multiple outputs, noise coupling to input or output, restricted duty cycle range, etc. $[4,5]$.

Some major constrains associated with the design and practical implementations are:

- Meeting the power demand. It is often difficult to deliver the required power to a load via ICWPT system due to limited space on the receiver side and specific power flow regulations.

- Switching speed. Operating at a higher frequency can help reduce the size of an ICWPT system. However, the switching speed of the switches is one of the major constraints. The most suitable switching devices for ICWPT applications seem to be insulated gate bipolar transistors (IGBTs) with commercial products up to the power level of $3 \mathrm{kV} / 2 \mathrm{kA}$, and a 
switching frequency up to $80 \mathrm{kHz}$. Power metal oxide silicon field effect transistors (MOSFETs) can switch at a speed up to MHz levels, but their voltage levels are too low for highpower ICWPT applications.

- Efficient operation. Due to various copper and ferrite losses, achieving high system power efficiency is a challenging task.

- Maximum temperature rise. This is an important factor to keep the system operating in an acceptable range of temperature, especially when the system is used in a specified temperature environment.

- System size/weight. The size and weight are limiting factors in designing an ICWPT system. The conversion process in power electronics requires the use of magnetic components that are usually the heaviest and bulkiest items of the circuit. The design of such components has an important influence on the overall system size/weight, power conversion efficiency, and cost.

- System stability and control. It is always important to have a stable system under full range of load and magnetic coupling variations in practical applications. Variable frequency controlled ICWPT power supplies can become unstable if not designed properly [6].

- Cost effectiveness. Existing ICWPT systems are costlier than traditional wired counterparts due to the complicated power electronic circuitry and magnetic coupling design. It is a challenging task to improve the system design to bring the cost down for practical applications.

- Compliance. Final practical products of ICWPT systems need to pass electromagnetic compatibility (EMC) and safety standards, which can be a challenging engineering design task.

The above mentioned challenges may interact with each other, making the system optimisation very difficult. Trade-offs often need to be made depending on practical constraints and requirements.

\section{Operating principle and general diagram of ICWPT systems}

\subsection{Operating principle}

The fundamental theory of ICWPT systems is governed by Faraday and Ampere's laws as shown in Figure 1. Based on Ampere's law, current I generates a magnetic field $H$. Some of this magnetic field links the secondary power pickup coil and according to Faraday's law causes a voltage $V$ to be induced.

Ampere's law can be mathematically expressed as:

$$
\oint \vec{H} \cdot \overrightarrow{d l}=I
$$


This equation states that the line integral of the magnetic field intensity around a closed loop is equal to the current flowing through it.

Faraday's law, on the other hand, is expressed by:

$$
V=-N_{2} \frac{d \varnothing}{d t}
$$

where $\mathrm{N}_{2}$ is the number of turns of the secondary coil.

The negative sign in (6) is described by Lenz's law, which states that the current flow in the secondary coil (when a load is connected) will be such that it creates a magnetic field that opposes the primary magnetic field.

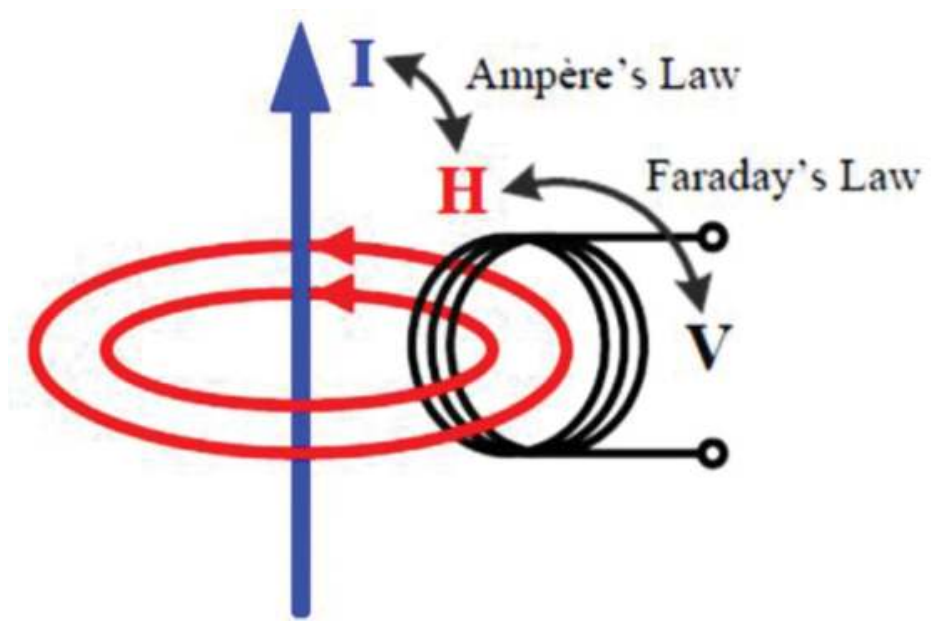

Figure 1. Fundamental theory of an ICWPT system.

\subsection{General block diagram of an ICWPT system}

A typical ICWPT system is normally constructed by winding the primary and secondary coils into separate halves of ferrite cores parted by an air gap as shown in Figure 2. Since direct physical contacts are eliminated in such a system, magnetic link is used to provide a reliable and efficient power transfer across an air gap. As it can be seen, the magnetic flux $\varphi_{\mathrm{m}}$ couples from one half of the core to the other half and provides a mutual inductance $M$ that couples energy from the primary to the secondary side. The mutual inductance $M$ is a function of geometry and can be found by simulation, measurement, or modelling the physical structure $[7,8]$. 


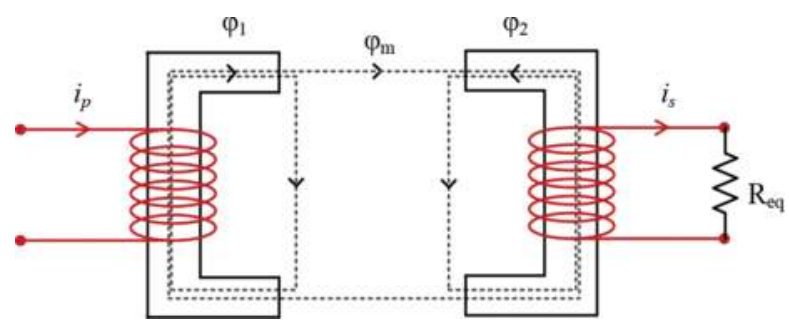

Figure 2. Typical flux linkage in an ICWPT system.

In such a loosely coupled power transfer system, the leakage fluxes can be very large and cannot be ignored. The proportion of the primary coils flux that links with the secondary coil is known as the primary coupling coefficient expressed by:

$$
k_{1}=\frac{\varnothing_{M}}{\varnothing_{1}}
$$

Likewise, the secondary coupling coefficient is given by:

$$
k_{2}=\frac{\varnothing_{M}}{\varnothing_{2}}
$$

These two values are typically combined to give the overall system coupling coefficient as:

$$
k=\sqrt{k_{1} k_{2}}
$$

The mutual inductance between the primary and the secondary coils then is given by:

$$
M=k \sqrt{L_{p} L_{s}}
$$

where $L_{p}$ and $L_{s}$ are the self-inductances of the primary and the secondary coils.

An ICWPT system can then be considered as a loosely coupled power transfer system using modern power conversion, control, and magnetic coupling techniques to achieve wireless power transfer as shown in Figure 3. It consists of a primary side AC/DC/AC resonant converter which converts the rectified AC power into high frequency AC power. The highfrequency AC power then is fed to the primary coil which is magnetically coupled to the secondary coil, while physically separated. The secondary side then can be movable (linearly or/and rotating), giving flexibility, mobility, and safeness for supplied loads. In the secondary side, the induced high-frequency power is converted and controlled by a secondary converter 
to meet the requirements specified by the load parameters. In fact, the time-varying magnetic field generated by the primary coil induces an electromotive force in the secondary coil which forms the voltage source of the secondary power supply. Since the magnetic coupling of an ICWPT system is normally very loose compared to normal transformers, the induced voltage source is usually unsuitable to be used to drive the load directly. Thus, a power conditioner with proper circuit tuning and conversion is required to control the output power according to the load requirements [9].

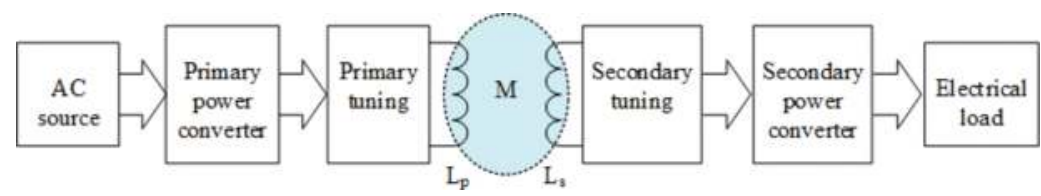

Figure 3. Fundamental elements of an ICWPT system.

\section{System tuning}

Tuning the primary and the secondary inductances of an ICWPT system is important for several reasons: (1) Compensating the primary inductance makes the power supply able to drive the given limited voltage ratings of the employed switches, (2) Constructing a resonant tank for resonant converters which is required to enable soft-switching operation of the switches to reduce switching losses and EMI, (3) Prevent the harmonic propagation in the circuit due to its filtering function, and (4) Compensating the secondary inductance improves the power transfer capability of the system.

\subsection{Primary side tuning}

Compensating the primary inductance improves the power factor and reduces the required Volt-Ampere rating of the primary power source. The compensation topologies are considered, namely series compensation, parallel compensation, and series-parallel compensation as shown in Figure 4. In series compensation, the voltage across the capacitance compensates the voltage drop of the primary equivalent reactance, making the required supply voltage to reduce. In parallel compensation, the current flowing through the capacitance compensates the current in the primary coil. Hence the required supply current is reduced. Series-parallel compensation realises the reduction of both in voltage and in current. In Figure 4 in a series compensation topology, the output current from the inverter bridge $\left(I_{1}\right)$ is equal to the primary current which is passing through the primary inductance $\left(I_{\mathrm{p}}\right)$. This signifies that the primary current in series compensation circulates through the inverter bridge which, in turn, causes significant power dissipation in the switching network. Moreover, in series compensation, the voltage is boosted because of the added voltage across the tuning capacitor. This results in an increased voltage across the primary coil $\left(V_{\mathrm{p}}\right)$ that is higher than the inverter output voltage $\left(V_{1}\right)$. This is beneficial as it allows the power supply to drive a high primary inductance with 
a desired primary current. The parallel compensation, on the other hand, has a current increase property. The primary current $\left(I_{\mathrm{p}}\right)$ is greater than the power converter output current $\left(I_{1}\right)$. This is because the reactive current is circulating inside the resonant tank, and only the real current is flowing through the inverter bridge. As a result, in a parallel tuned power supply design, lower current rated switches may be used. The value of the primary compensation capacitor is often designed to fully compensate the primary inductance at the primary operating angular frequency $(\omega)$. In this case, the primary compensation capacitance is determined by:

$$
C_{p}=\frac{1}{\omega^{2} L_{p}}
$$
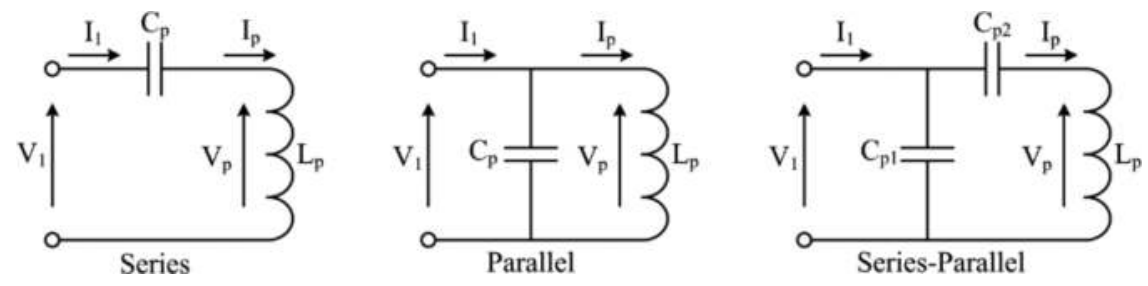

Figure 4. Primary tuning topologies.

Note that the capacitance of (7) is calculated for either a series- or a parallel-tuning topology. In the case of a composite topology, it is a combination of the two topologies. One simple way is to use $C_{p 2}$ to fully cancel the primary leakage inductance $\left(L_{p}-M\right)$, and $C_{p 1}$ to fully tune the remaining portion of the primary self-inductance $(M)$ :

$$
\begin{gathered}
C_{p 1}=\frac{1}{\omega^{2} M} \\
C_{p 2}=\frac{1}{\omega^{2}\left(L_{p}-M\right)}
\end{gathered}
$$

\subsection{Secondary side tuning}

In an ICWPT system, because of the large internal reactance, the power transferred from the primary to the secondary side is not sufficient for the load. For an uncompensated secondary inductance, the maximum output power occurs when the load resistance $R_{L}$ is equal to the internal reactance $\omega L_{s}$, and can be expressed by [9]:

$$
P_{o . \max }=\frac{1}{2} V_{o c} I_{s c}
$$


where $V_{o c}$ is the open-circuit voltage at the secondary terminals and $I_{s c}$ is the short-circuit current of the secondary side.

In order to improve the power transfer capability of the system, normally the secondary side is tuned to the primary side operating frequency. There are two basic tuning topologies used in the secondary side, namely series compensation and parallel compensation as shown in Figure 5. An equivalent resistance $\left(R_{e q}\right)$ represents the equivalent load on the secondary side.

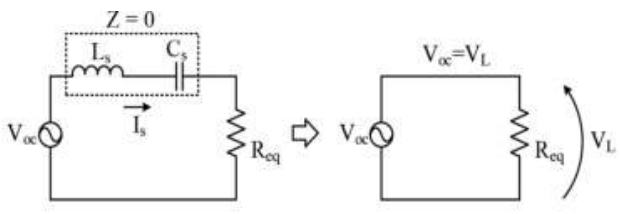

(a) Series tuning

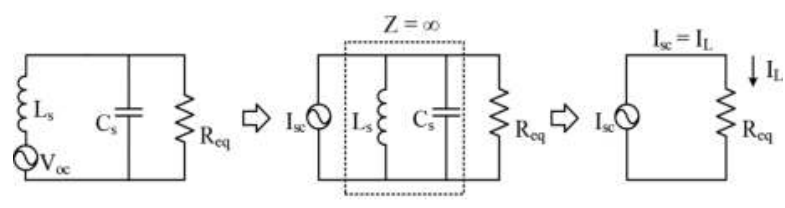

(b) Parallel tuning

Figure 5. Secondary side-tuning topologies. (a) Series tuning. (b) Parallel tuning.

In Figure 5(a); the equivalent reactance and its compensation capacitance cancel each other at the resonant frequency, making the output voltage independent of the load and equal to the secondary open-circuit voltage. Likewise, in parallel compensation the equivalent admittance and its compensating capacitance cancel each other at the resonant frequency, which makes the output current independent of the load and equal to the secondary short circuit current as illustrated in Figure 5(b). For maximum power transfer, the secondary resonant frequency is normally designed to equal the nominal frequency. In this case, the secondary compensation capacitance is determined as:

$$
C_{s}=\frac{1}{\omega^{2} L_{s}}
$$

\section{Equivalent circuit and power transfer capability}

\subsection{T-equivalent circuit}

Typical ICWPT systems are modelled using the conventional transformer model with an equivalent T-circuit as shown in Figure 6. It should be noted that normally the air gap in the path of the flux leads to low flux densities which tend to make the core losses very small, so 
the core losses component may be neglected from the model without significant loss of accuracy.

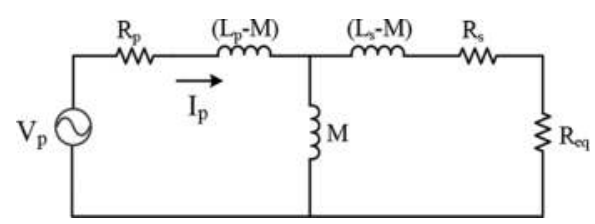

Figure 6. T-equivalent circuit.

From the above model, the power transfer capability of an ICWPT system is determined by two fundamental parameters: $V_{o c}$ being the open-circuit voltage (when $R_{e q}=\infty$ ), and $I_{s c}$ being the short-circuit current (when $R_{e q}=0$ ) obtained from two basic open-circuit and short-circuit tests as [10]:

$$
\begin{gathered}
V_{o c}=j \omega M I_{p} \\
I_{s c}=I_{p} \cdot \frac{j \omega M}{R_{s}+j \omega\left[M+\left(L_{s}-M\right)\right]}
\end{gathered}
$$

In practice, in order to reduce the coils losses, a woven Litz wire is normally used with low ESR (Equivalent Series Resistance); therefore, in (13) $R_{s}$ can be neglected giving the short-circuit current as [10]:

$$
I_{s c}=I_{p} \cdot \frac{M}{L_{s}}
$$

From the above equations, the uncompensated power $S_{u}$ of the secondary side can be expressed as follows [10]:

$$
S_{u}=V_{o c} I_{s c}=\omega \cdot \frac{M^{2}}{L_{s}} \cdot I_{p}^{2}
$$

In practice, the maximum power transferred to the load side without compensation is $S_{u} / 2$. The power presented by (15) can be boosted up $Q_{S}$ times, using resonant techniques. As detailed in the previous section, a capacitance can be added to the circuit in series or parallel to tune the secondary inductance to the primary operating switching frequency. The maximum power transferred to the load side for a certain $Q_{S}$ then can be given as follows: 


$$
P_{2}=Q_{s} S_{u}=Q_{s} \cdot \omega \cdot \frac{M^{2}}{L_{s}} \cdot I_{p}^{2}
$$

where $Q_{S}$ is the secondary circuit quality factor and it is different depending on the secondary tuning topology as given by:

$$
Q_{s}=\left\{\begin{array}{l}
\text { Series }- \text { tuned secondary }: \frac{\omega L_{s}}{R_{e q}} \\
\text { Parallel }- \text { tuned secondary }: \frac{R_{e q}}{\omega L_{s}}
\end{array}\right.
$$

It should be noted that for the parallel-tuned secondary, the secondary coil appears as a current source, while for a series-tuned secondary it acts as a voltage source. The tuned output of the secondary coil is then rectified and regulated to a constant output voltage using an appropriate switch-mode controller according to the load requirements. However, based on the tuning topology, different power regulation circuitry such as boost, buck, etc. is used on the secondary side [11]. The quality factor $Q s$ is determined by the secondary circuit parameters as well as the equivalent output load, which is related to the operation of the switch-mode controller. It is an important parameter which needs to be chosen carefully during design. Although higher $Q_{S}$ increases the power transfer capability, for several reasons in practice, the highest $Q_{S}$ reachable is limited to about 10. Firstly, the secondary operational bandwidth is inversely proportional to $Q_{S}$. Secondly, higher $Q_{S}$ would be obtained at the expenses of the higher secondary VA rating so that practical size and thermal ratings also naturally limit $Q_{S}$ in commercial systems. Thirdly, practical component tolerances and aging affect the sensitivity of the circuit and make power transfer at high $Q_{S}$ impractical.

\subsection{Mutual inductance model}

The mutual inductance model uses the concepts of induced and reflected voltages to describe the coupling effect between the secondary and the primary networks. Both the induced and the reflected voltages are expressed in terms of the mutual inductance as shown in Figure 7. Note that coils losses are assumed to be very small and not considered in the presented model. In a mutual inductance model, the leakage inductance is not modelled separately from the mutual portion for circuit analysis as in other models such as T-equivalent circuit. This is a major advantage for ICWPT systems where the leakage inductance is too large to be ignored.

In the mutual inductance model, the reflected voltage is used to represent the total effect of the secondary on the primary side. However, the effect of the secondary must be considered together with the inherent properties of the primary coil in the analysis of the primary network. The reflected voltage is determined by the current flowing within the secondary coil and the mutual inductance between the primary and the secondary as given by: 


$$
V_{r}=-j \omega M I_{s}
$$

The effect of the secondary can be represented by the equivalent reflected impedance by dividing the reflected voltage by the primary current as follows:

$$
Z_{r}=\frac{V_{r}}{I_{p}}=\frac{-j \omega M I_{s}}{I_{p}}
$$

The secondary current flowing through the secondary coil $\left(I_{s}\right)$, on the other hand, is defined by the input impedance seen by the open circuit voltage as:

$$
I_{s}=\frac{V_{o c}}{Z_{s}}=\frac{j \omega M I_{p}}{Z_{s}}
$$

Combining (19) and (20), the equivalent reflected impedance of the secondary back on to the primary is expressed as:

$$
Z_{r}=\frac{\omega^{2} M^{2}}{Z_{s}}
$$

The overall impedance of the secondary side for both series and parallel compensated secondary side can be expressed as:

$$
\begin{gathered}
Z_{s}(\text { series })=j \omega L_{s}+\frac{1}{j \omega C_{s}}+R_{e q} \\
Z_{s}(\text { parallel })=j \omega L_{s}+\frac{1}{j \omega C_{s}+\frac{1}{R_{e q}}}
\end{gathered}
$$

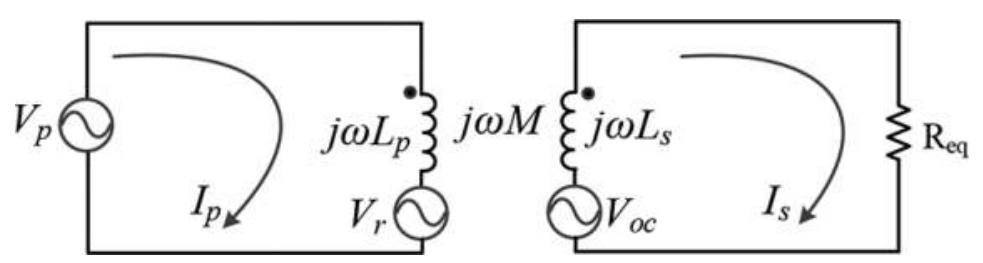

Figure 7. Frequency domain mutual inductance model. 
Since the secondary network depends on the selected tuning topology, the reflected resistance and reactance at the resonant frequency onto the primary side are different as given in Table 1.

\begin{tabular}{lll}
\hline Tuning topology & Reflected resistance & Reflected reactance \\
\hline Series & $\frac{\omega^{2} M^{2}}{R_{e q}}$ & 0 \\
Parallel & $\frac{M^{2} R_{e q}}{L_{s}^{2}}$ & $-\frac{\omega M^{2}}{L_{s}}$ \\
\hline
\end{tabular}

Table 1. Reflected impedance for different secondary tuning.

It can be seen from Table 1 that the reflected impedance for the series-tuned secondary coil is purely resistive, while the parallel-tuned secondary coil reflects both resistive and capacitive components. This is one of the major differences between the series and the parallel compensated secondary inductance. The reflected impedance can then be compared and combined with the impedance of the primary coil. The power transferred from the primary to the secondary is simply the reflected resistance (real part of the reflected impedance) multiplied by the squared primary current as given by:

$$
\begin{gathered}
P_{2}(\text { seriestuning })=\left[\frac{\omega^{2} \mathrm{M}^{2}}{\mathrm{R}_{\mathrm{eq}}}\right] I_{p}^{2} \\
P_{2}(\text { parallel tuning })=\left[\frac{\mathrm{M}^{2} \mathrm{R}_{\mathrm{eq}}}{\mathrm{L}_{\mathrm{s}}^{2}}\right] I_{p}^{2}
\end{gathered}
$$

\section{Power losses associated with the magnetic structure of ICWPT systems}

Two types of power losses are associated with the magnetic coupling structure of an ICWPT system: (1) core losses and (2) coils losses (conduction losses) as detailed below:

\subsection{Core losses}

Typically, core losses are divided into two groups: hysteresis and eddy current losses. Hysteresis loss increases with higher frequencies as more cycles are undergone per unit time and is associated with the applied voltage and its frequency. While, eddy currents loss is proportional to the square of the applied voltage and it is independent of frequency. In the case of core losses due to magnetic hysteresis, all ferromagnetic materials tend to retain some degree of magnetisation after disclosure to an external magnetic field. This tendency to stay magnetised is called hysteresis. And it takes a certain amount of energy to overcome this 
opposition to change every time when the magnetic field produced by the primary coil changes polarity (twice per AC cycle). This type of loss can be mitigated by choosing a core with low hysteresis (with a 'thin' B-H curve), and designing the core for minimum flux density (large cross-sectional area). The hysteresis losses per unit volume are approximated by the following relationship [12]:

$$
P_{h}=k f_{s w}^{a}\left(\frac{\Delta B}{2}\right)^{d} \quad\left(W / m^{3}\right)
$$

where $k, a$, and $d$ are the material-related constants, $f_{s w}$ is the switching frequency, and $\Delta B$ is the flux excursion.

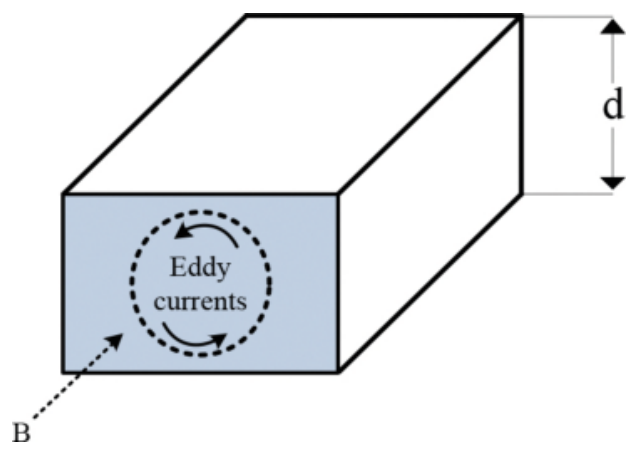

Figure 8. Eddy currents created in a magnetic material.

The second part of the core losses is due to magnetic effects of the core material. This type is resistive power dissipation due to the induced eddy currents in the core material. Usually, when a low resistivity material is placed in a variable magnetic field, electric currents are induced in the material similar to the currents induced in the secondary coil. These induced currents tend to circulate through the cross-section of the core perpendicular to the main flux path. Their circular motion gives them their unusual name: like eddies in a stream of water that circulates rather than move in straight lines. These 'eddy currents' must overcome an electrical resistance as they circulate in the core. To overcome the resistance offered by the core, they dissipate power in the form of heat. Hence, there will be a source of power loss in the core that is difficult to eliminate and largely depends on the conductivity of the material and the magnetic field density. Thus, eddy currents can be minimised by selecting magnetic core materials that have low electrical conductivity. Ferromagnetic materials such as ferrites have a considerably low electrical conductivity, making them more suitable for high-frequency applications. The eddy current losses in a volume like that of Figure 8 are approximated by the following relationship [12]: 


$$
P_{e}=\frac{B^{2} f_{s w}^{2} d^{2}}{\rho} \quad\left(W / m^{3}\right)
$$

where $\rho$ is the resistivity of the material and $f_{s w}$ is the switching frequency.

\subsection{Conduction losses}

In practice, there will always be power dissipated in the form of heat through the resistance of current-carrying conductors. Increasing the gauge of the wire can minimise this loss, but only with extensive increases in cost, size, and weight. Generally, the conduction losses of the coils are classified into two groups: (1) the DC and (2) the AC losses. When a DC current flows through an inductor, normally DC losses are the result of current $I$ flowing through the wire with resistance $R$, power $\left(I^{2} R\right)$ is dissipated and, accordingly, developing heat in the wire. When an inductor is used in an AC circuit, on the other hand, normally due to the varying magnetic field around the coils, the current distribution in the conductors is not uniform. The selfinductance of the conductor, as well as the magnetic field created by adjacent turns of the same coil, redistributes the current flow within the wire and reduces the effective cross-sectional area of the conductor and accordingly increases the AC resistance. These effects are known as skin and the proximity effects, and they become stronger at higher operating frequencies as detailed below.

\subsubsection{The skin effect}

When a wire is carrying a DC current, the current is distributed uniformly over all the effective area of the wire. This current distribution is non-uniform when the wire is carrying an alternating current, as the current intensity and the magnetic field in a conductor change and results in a phenomenon known as 'skin effect'. The skin effect is the restriction of the flow of alternating current to the surface of a conductor. This restriction is caused by the alternating magnetic field that the current itself generates within the conductor. As depicted in Figure 9(a), the current flowing in the conductor generates a magnetic field around it. This field in turn creates circulating currents in the wire. These currents are opposite to the main current flow in the centre of the conductor and add to the main current closer to the surface of the wire. The result is that more net current is flowing closer to the surface of the conductor, and eventually an increase in the effective resistance of the conductor due to the reduced crosssectional area for current flow as shown in Figure 9(b). This area of current flow between the outer surface and a level of the conductor diameter is called the 'skin depth'. The skin depth is thus defined as the depth below the surface of the conductor at which the current density has fallen to $1 / e$ (about 0.37 ) of $J_{S}$. Typically, it is well approximated as:

$$
\delta=\sqrt{\frac{2 \rho}{\omega \mu}}
$$


where $\rho$ is the resistivityof the conductor, $\omega$ is the angular frequencyof the current, and $\mu=$ $\mu_{r} \mu_{0}$ is the absolute magnetic permeability of the conductor.

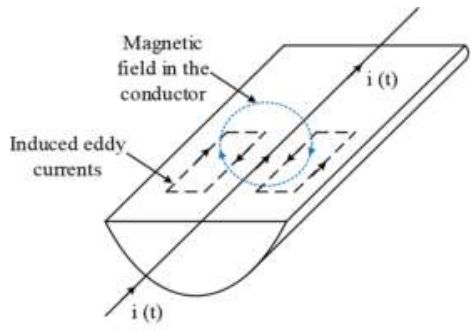

(a)

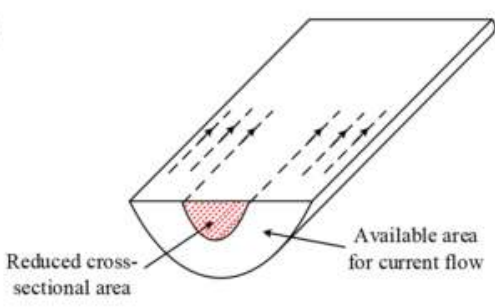

(b)

Figure 9. Impact of skin effect on the coil ESR: (a) generated eddy currents and (b) reduced cross-sectional area due to skin effect.

\subsubsection{The proximity effect}

Proximity effect is the tendency for current to flow in other undesirable patterns, loops, or concentrated distributions due to the presence of magnetic fields generated by nearby conductors as shown in Figure 10(a). In fact, when another conductor is brought into proximity to one or more other nearby conductors, such as within a closely wound coil of wire, the distribution of current within the conductor will be constrained to smaller regions as shown in Figure 10(b) in blue. Field intensity is no longer uniform around each conductor surface. The resulting current crowding is termed as the 'proximity effect' and increases with the frequency. This current crowding gives an increase in the effective resistance which results in higher power losses in the wire.

In transformers and inductors, proximity-effect losses typically dominate over skin-effect losses [13]. In Litz-wire windings, proximity effect may be further divided into internal proximity effect (the effect of other currents within the bundle) and external proximity effect (the effect of current in other bundles). The actual losses in one strand of a Litz bundle are

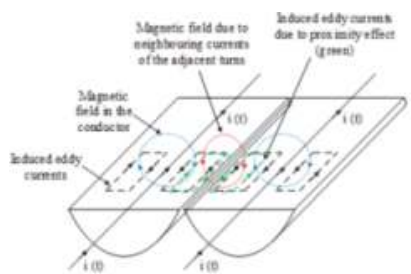

(a)

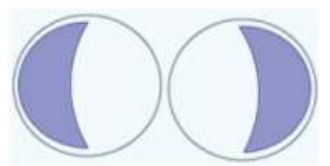

(b)

Figure 10. Two conductors in proximity: (a) magnetic fields generated by nearby conductors and (b) impact of proximity effect on the cross-sectional area of a conductor. 
simply a result of the total external field, due to the currents in all the other strands present. One approach of calculating the loss in a Litz winding is to look at it as a single winding, made up of $n N$ turns of the strand wire, each with current $i / n$ flowing in it, where $n$ is the number of strands, $N$ is the number of turns of Litz wire, and $i$ is current flowing in the overall Litz bundle. The loss in the Litz winding will be the same as in the equivalent single-strand winding as long as the currents flowing in all the strands are equal [13].

\section{System magnetic coupling efficiency}

\subsection{Series-tuned secondary}

Figure 11 shows a diagram of a magnetic coupling structure of two magnetically coupled coils. The inductances $L_{p}$ and $L_{s}$ represent the primary and the secondary coils, and $R_{e q}$ represents the equivalent load resistance which includes the DC load resistance and any other resistive part of the circuit such as the rectifier, voltage regulator, etc.

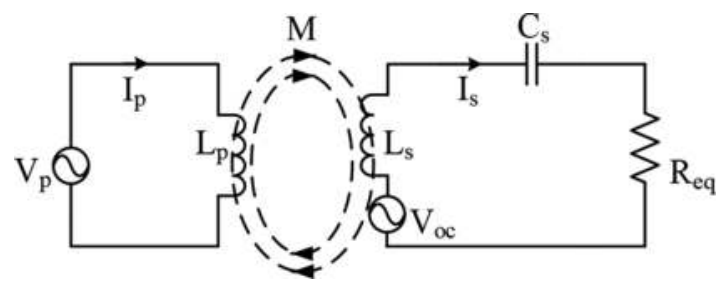

Figure 11. Simplified circuit with a series-tuned secondary.

The coupling efficiency of the circuit shown in Figure 11 can be simply expressed by:

$$
\begin{aligned}
& \eta_{k}=\frac{\text { output power across } R_{e q}}{\text { output power across } R_{e q}+\text { power losses }} \\
& =\frac{P_{e q}}{P_{e q}+P_{\text {loss } 1}+P_{\text {loss } 2}}
\end{aligned}
$$

where $P_{\text {loss } 1}$ and $P_{\text {loss2 }}$ are the primary and the secondary coils power losses respectively, and $P_{e q}$ is the supplied active power to the load.

The power losses of the primary and secondary coils can be quantified based on their unloaded and loaded quality factors. The unloaded quality factors are basically the coils quality factors $\left(Q_{L p} \& Q_{L s}\right)$; and when a load is added, it is referred to as the loaded or circuit quality factor $\left(Q_{s} \& Q_{p}\right)$. The general definition of the quality factor is the ratio of stored energy in the circuit to the energy lost in the circuit as given by: 


$$
\begin{aligned}
& Q=\frac{\text { Stored energy }}{\text { Dissipated energy }}=\frac{\text { Reactive power }}{\text { Active power }} \\
& =\frac{V A R}{W A T T}=\frac{\omega L}{R}
\end{aligned}
$$

From the above definition, the primary and the secondary coils quality factors are:

$$
\begin{gathered}
Q_{L p}=\frac{V A R_{p}}{P_{\text {loss } 1}}=\frac{\omega L_{p}}{R_{p}} \\
Q_{L s}=\frac{V A R_{s}}{P_{\text {loss } 2}}=\frac{\omega L_{s}}{R_{s}}
\end{gathered}
$$

From (31) and (32), the power losses of the primary and secondary coils can be written as:

$$
\begin{gathered}
P_{\text {loss } 1}=\frac{V A R_{p}}{Q_{L p}} \\
P_{\text {loss } 2}=\frac{V A R_{s}}{Q_{L s}}
\end{gathered}
$$

Under load conditions, the secondary circuit quality factor is:

$$
Q_{s}=\frac{V A R_{s}}{P_{l o s s 2}+P_{e q}}
$$

From (35), the secondary reactive power can then be expressed as:

$$
V A R_{s}=\left(P_{\text {loss } 2}+P_{e q}\right) Q_{s}
$$

Substituting (36) into (34):

$$
P_{\text {loss } 2}=\frac{P_{e q} \frac{Q_{s}}{Q_{L s}}}{\left(1-\frac{Q_{s}}{Q_{L s}}\right)}=\frac{P_{e q} Q_{s}}{Q_{L s}-Q_{s}}
$$


The primary reactive power can also be expressed in terms of the secondary circuit quality factor as [14]:

$$
V A R_{p}=\frac{P_{e q}}{k^{2} Q_{s}}
$$

Substituting (38) into (33), the primary coil power loss can be rewritten as:

$$
P_{l o s s 1}=\frac{P_{e q}}{k^{2} Q_{s} Q_{L p}}
$$

Substituting (37) and (39) into (29) gives the coupling efficiency for a series-tuned secondary as:

$$
\begin{aligned}
& \eta_{k}=\frac{P_{e q}}{P_{e q}+\frac{P_{e q}}{k^{2} Q_{s} Q_{L p}}+\frac{P_{e q} Q_{s}}{Q_{L s}-Q_{s}}} \\
& =\frac{1}{1+\frac{1}{k^{2} Q_{s} Q_{L p}}+\frac{Q_{s}}{Q_{L s}-Q_{s}}}
\end{aligned}
$$

If $Q_{L S} \gg Q s$, the term, $Q_{L s}-Q_{s} \approx Q_{L s}$. Hence:

$$
\eta_{k}=\frac{1}{1+\frac{1}{k^{2} Q_{s} Q_{L p}}+\frac{Q_{s}}{Q_{L s}}}
$$

\subsection{Parallel-tuned secondary}

A parallel-tuned secondary coil shown in Figure 12 is in fact a Norton equivalent of the seriestuned secondary coil.

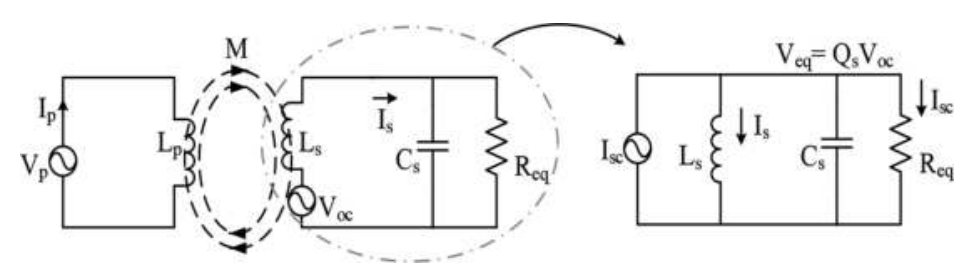

Figure 12. Simplified circuit with a parallel-tuned secondary. 
Similar to the series-tuned secondary, the power losses within the primary and the secondary coils can be expressed in terms of the coils quality factors as:

$$
\begin{gathered}
P_{\text {loss } 1}=\frac{V A R_{p}}{Q_{L p}} \\
P_{\text {loss } 2}=\frac{V A R_{s}}{Q_{L s}}
\end{gathered}
$$

The output power across $R_{e q}$ is:

$$
P_{e q}=I_{s c}^{2} R_{e q}
$$

In a parallel-tuned secondary, the resonant current through the coil is:

$$
I_{s}=I_{s c} \sqrt{1+Q_{s}^{2}}
$$

The reactive power of the secondary coil then is:

$$
\begin{aligned}
& V A R_{s}=I_{s}^{2} \omega L_{s}=\left[I_{s c} \sqrt{1+Q_{s}^{2}}\right]^{2} \omega L_{s} \\
& =I_{s c}^{2}\left(1+Q_{s}^{2}\right) \omega L_{s}
\end{aligned}
$$

Taking the ratio of (46) and (44):

$$
\frac{V A R_{s}}{P_{e q}}=\frac{\left(1+Q_{s}^{2}\right) \omega L_{s}}{R_{e q}}=\frac{\left(1+Q_{s}^{2}\right)}{Q_{s}}
$$

Substituting VARs from (47) into (43):

$$
P_{l o s s 2}=P_{e q} \frac{\frac{\left(1+Q_{s}^{2}\right)}{Q_{s}}}{Q_{L s}}=P_{e q} \frac{\left(1+Q_{s}^{2}\right)}{Q_{s} Q_{L s}}
$$

Again the primary reactive power can also be expressed in terms of the secondary circuit quality factor as: 


$$
V A R_{p}=\frac{P_{e q}}{k^{2} Q_{s}}
$$

Substituting (49) into (42), the primary coil power loss can be rewritten as:

$$
P_{l o s s}=\frac{P_{e q}}{k^{2} Q_{s} Q_{L p}}
$$

Substituting (48) and (50) into (29) gives the coupling efficiency for a parallel-tuned secondary as:

$$
\eta_{k}=\frac{1}{1+\frac{1}{k^{2} Q_{s} Q_{L p}}+\frac{\left(1+Q_{s}^{2}\right)}{Q_{s} Q_{L s}}}
$$

If $Q_{s}^{2} \gg 1,(51)$ becomes:

$$
\eta_{k}=\frac{1}{1+\frac{1}{k^{2} Q_{s} Q_{L p}}+\frac{Q_{s}}{Q_{L s}}}
$$

\section{Summary}

This chapter has presented the fundamentals of ICWPT systems. Their major application areas, operating principle, and present challenges associated with this technology have been discussed. This technology has the ability to deliver major advancements in industries and applications that are dependent on contacting connectors, which can be unreliable and prone to failure. The basis of a wireless power transfer system involves essentially two magnetically coupled coils separated by an air gap - a transmitter and a receiver coil. The transmitter coil is energised by alternating AC power to generate a magnetic field, which in turn induces a voltage in the receiver coil.

After a general overview of system compensation in this technology, a detailed modelling based on T-equivalent circuit as well as a mutual inductance model including system power transfer capability have been presented. Power losses associated with the magnetic coupling structure of such a system have been detailed. A comprehensive study with mathematical relationships has been conducted on the derivation of magnetic coupling efficiency of the system for both a series- and a parallel-tuned secondary side. 


\title{
Author details
}

\author{
Ali Abdolkhani
}

Address all correspondence to: aabd104@aucklanduni.ac.nz

Power by Proxi Ltd, Auckland, New Zealand

\section{References}

[1] C. Fernandez, O. Garcia, R. Prieto, J. Cobos, and J. Uceda, “Overview of different alternatives for the contact-less transmission of energy," IECON (Industrial Electronics Society, IEEE 2002 28th Annual Conference), 2002, vol. 2, pp. 1318-1323.

[2] J. Boys, G. Covic, and A. W. Green, "Stability and control of inductively coupled power 16 transfer systems," IEE Proceedings - Electric Power Applications, 2000, vol. 147, pp. 37-43.

[3] M. S. Rauls, D. W. Novotny, and D. M. Divan, "Design considerations for highfrequency coaxial winding power transformers," IEEE Transactions on Industry Applications, 2002, vol. 29, pp. 375-381.

[4] R. W. Erickson and D. Maksimovi, Fundamentals of Power Electronics, Springer, The Netherlands, 2001.

[5] M. H. Rashid, Power Electronics Handbook, Academic Press, 2001.

[6] T. Chunsen, D. Xin, W. Zhihui, S. Yue, and A. P. Hu, "Frequency bifurcation phenomenon study of a soft switched push-pull contactless power transfer system," 6th IEEE Conference on Industrial Electronics and Applications, 2011, pp. 1981-1986.

[7] A. Abdolkhani, A. P. Hu, and N. C. K. Nair, "Modelling and parameters identification of through-hole type wind turbine contactless sliprings," SCIRP/Engineering, 2012, vol. 4, pp. 272-283.

[8] K. D. Papastergiou and D. E. Macpherson, "Contact-less transfer of energy by means of a rotating transformer," Proceedings of the IEEE International Symposium on Industrial Electronics, 2005. ISIE 2005, 2005, pp. 1735-1740.

[9] A. P. Hu, "Selected resonant converters for IPT power supplies," PhD Thesis, University of Auckland, 2001.

[10] S. Raabe, G. A. J. Elliott, G. A. Covic, and J. T. Boys, “A quadrature pickup for inductive power transfer systems," 2nd IEEE Conference on Industrial Electronics and Applications, 2007. ICIEA 2007, 2007, pp. 68-73. 
[11] H. H. Wu, J. T. Boys, and G. A. Covic, "An AC processing pickup for IPT systems," IEEE Transactions on Power Electronics, 2010, vol. 25, pp. 1275-1284.

[12] N. Mohan, T. M. Undeland, and W. Robbins, "Power Electronics: Converters, Applications and Design, 1995, John Wiley and Sons, New York, 1997.

[13] C. R. Sullivan, “Optimal choice for number of strands in a litz-wire transformer winding," IEEE Transactions on Power Electronics, 1999, vol. 14, pp. 283-291.

[14] G. A. Covic and J. T. Boys, "Inductive power transfer," Proceedings of the IEEE, 2013, vol. 101, pp. 1276-1289. 
